to the genetics of shell coiling in some gastropods, in which the direction of coiling is determined by a single Mendelian locus with two alleles, and homeotic mutants in Drosophila. And while one cannot deny the importance of these systems to an understanding of developmental mechanics, it is not immediately obvious why they represent the same mechanism by which novel phenotypes arise.

Arthur's downplaying of developmental mechanics stems from his belief that the developmental origin of "saltational variants" is well established and not, therefore, a problem; the real problem, he offers, "occurs at the level of the population rather than at that of the individual" (p. 179). This should come as a surprise to many evolutionary biologists. For instance, Futuyma (1979 p. 438) states: "The real problems posed by evolution . . . lie not so much in the potency of natural selection as in the mechanisms by which the variations on which it acts arise .... The problem of how new variations arise falls not within the province of mathematical genetical theory, but within that of molecular genetics and developmental biology." The problem, as Arthur sees it, stems from the fact that his saltational variants are not perfect (this would be asking too much of the developmental system); they must be sustained through a maladaptive phase lasting several generations until numerous minor mutations bring the novel structure, or body plan, to a new adaptive peak. Thus, we are introduced to the concept of "n-selection"-a regime in which an organism's survival seemingly is based solely on whether or not it can breed successfully. As this regime entails independence from biotic factors such as competition, the mutation involving a $D$-gene is therefore also assumed to provide "ecological and reproductive isolation from the wild-type progenitor" (p. 188) in addition to morphological novelty. Saltation indeed.

The main strength of Mechanisms of Morphological Evolution is its primary focus on a problem that typically is relegated to a subsidiary role in general treatments of evolutionary biology: how do morphological differences at the level of order, class, or even phylum arise and become established? Arthur does a good job of pointing out aspects of this problem that lack convincing explanations derived from the Modern Synthesis. He also usefully calls attention to the necessity of incorporating population-level phenomena in what traditionally has been considered from a narrow morphological perspective. As an alternative, however, he offers a saltational model that is, at least with respect to certain aspects of genetics, development, and ecology, less realistic than almost any scenario provided by the synthetic view. The answer to this problem must lie between these two extremes, but determination of exactly where the answer is still seems far off.

\section{Literature Cited}

FuTUYMA, D. J. 1979. Evolutionary Biology. Sinauer, Sunderland, MA.

MAYR, E. 1963. Animal Species and Evolution. Harvard Univ. Press, Cambridge, MA.

Corresponding Editor: J. B. Mitton

\title{
THE NEOLITHIC TRANSITION AND THE GENETICS OF POPULATIONS IN EUROPE:
} A REVIEW ${ }^{1}$

\author{
Peter E. Smouse \\ Department of Human Genetics and Division of Biological Sciences \\ University of Michigan \\ Ann Arbor, MI 48109
}

Received October 14, 1985

One of the more engrossing pastimes that has occupied human geneticists over the last 40 years has been the attempt to make some evolutionary sense out of the patterns of genetic variation that typify the species Homo sapiens. The first lesson we have learned from this sort of work is that it is seldom possible to infer the details of evolutionary history from the genetic data alone; there are simply too many factors that have impinged on genetic variation in species to permit unambiguous inference as to causation and timing. The

\footnotetext{
1 The Neolithic Transition and the Genetics of Populations in Europe. A. J. Ammerman and L. L. CavalliSforza. Princeton Univ. Press, Princeton, NJ, 1984. xv +176 pp. $\$ 25.00$.
}

second lesson we have learned is that the more information we have from extraneous (non-genetic) sources, the better job of evolutionary reconstruction we can do. What this means in practice is that we use the genetic data not so much to infer human history as to confirm it. In The Neolithic Transition and the Genetics of Populations in Europe, an archaeologist (A. J. Ammerman) and a population geneticist (L. L. Cavalli-Sforza) use the available evidence from genetic marker frequencies to support their claim that early agriculture spread from southwest Asia across Europe, effecting the neolithic transition in the process.

The book begins with a brief description of the origins of agriculture in southwest Asia. A distinction is drawn between the initial development of domestic plant and animal species from wild progenitors and the incor- 
poration of these species into a well developed agricultural system. The domestication phase seems to have occurred between 10,000 and 9,000 BP in southwest Asia, whereas full blown agriculture was clearly in evidence by 8,000 to $7,000 \mathrm{BP}$.

The neolithic transition in Europe has traditionally been defined in terms of tool-crafting techniques and pottery styles. More recently, emphasis has shifted to the types of subsistence economies represented by different types of tool manufacture. The authors take the position that the neolithic transition defined in terms of tools and pottery reflects the development of an agricultural economy throughout Europe; evidence exists to suggest that this agricultural system was introduced from outside, rather than being developed in situ and de novo.

Archaeological evidence and radio carbon dating suggest that agriculture spread across Europe in a generally northwesterly direction at the rate of about 1 $\mathrm{km} / \mathrm{yr}$ (or $25 \mathrm{~km} /$ generation). The total process is thought to have taken 2,500 to 3,000 years. The central question addressed in the book is whether that spreading process was strictly a matter of cultural (innovation) diffusion, with agricultural techniques being passed from one group to the next, or whether it was the farmers themselves who moved. The authors argue that, in large part, it was the farmers themselves who moved, and term this process "demic diffusion."

Their argument is based on the assumption that cultural diffusion and demic diffusion would have different consequences for the pattern of genetic divergence among current day populations in Europe. Under this assumption, extant genetic patterns should provide information on the processes involved in the neolithic transition. Given cultural transmission, there should be no long-range genetic continuity, and, barring the patchy cohesion expected from localized gene flow, there should be no larger regional trends. Given demic diffusion, however, there should exist a directional cline in allele frequencies running roughly southeast to northwest along an axis paralleling the spread of agriculture.

Using R. A. Fisher's model for a wave of advance, they show how genes from southwest Asia would have swept across Europe. Although the farmers, having a much larger subsistence base, would have greatly outreproduced their mesolithic neighbors, a certain amount of gene flow would have occurred between the two populations as the outnumbered hunter-gatherers became assimilated into the agricultural society. Over 2,500 to 3,000 miles, the net effect would have been a gradual diminution of the genetic impact of the original farmers from southwest Asia, thus creating a cline.

The authors substantiate the southeast to northwest cline expected from the demic diffusion model by using principal components analysis on the frequencies of 39 alleles, a large number of them from the HLA complex. Remarkably, this cline accounts for approximately $30 \%$ of the genetic variation observed among modern populations. They also extract an additional pair of principal components that together account for a further $30 \%$ of the variation. The first runs roughly east and west and the second spreads in all directions from a focal point north of the Black Sea. They tentatively postulate large scale gene flow from the east that postdates the neolithic transition as an explanation of the east-to-west cline, and point out that the focal zone of the third component coincides with the purported source region for the radiation of Indo-European languages (and presumably people). Neither matter is pursued further, being left for later work. They briefly present the results of some rather extensive computer simulations on the processes involved and show that the model hangs together rather nicely.

They are careful to point out that the genetic data are compatible with the demic diffusion hypothesis, rather than proving it. While it is possible to imagine other population processes that might have produced similar genetic results, they have nevertheless made a good circumstantial case for the demic diffusion model. Future evidence may or may not support their view. Regardless of the ultimate fate of their model, however, the book will remain a lovely demonstration of what can be accomplished when experts from different fields pool their intellectual resources.

The book represents a synthesis of more than a decade's collaboration between Ammerman and CavalliSforza. The presentation is nicely crafted, simple and coherent, with the esoteric technical details of archaeology, population genetics, and statistics either illustrated heuristically or referenced. It is an enjoyable Sunday afternoon read, yet contains some pithy material. I recommend it heartily.

Corresponding Editor: J. B. Mitton 